\title{
Ist der periodische Verlauf des gastro-duodenalen Ulcus
} saisongebunden?

Will man die Pathogenese des gastro-duodenalen Ulcus in eine ein-fache Formel bringen, so kann man wohl sagen, daß es sich um eine Störung im Gleichgewicht zwischen peptischer Aktivität des Magen-saftes und den, die Autodigestion verhindernden Schutzmechanismen in der Magenwand handelt. Eine solche Gleichgewichtsstörung wird bei wochenlanger Medikation von Corticotropin oder Cortison be-obachtet. Dabei läßt sich die gesteigerte peptische Aktivität an stei-genden Säurewerten und vermehrter Pepsinproduktion im Magen-saft [1, 2] und vermehrter Uropepsinausscheidung im Urin [3, 4, 5] nachweisen. Gleichzeitig kommt es zu einer Abnahme der Gewebe-

52

\section{Editorial}

resistenz mit vaskulär trophischen Störungen [6], mangelhaftem Funktionieren der die Magenschleimhaut überziehenden «Mucus-barriere» [7, 8] und endlich zur Hemmung reparativer Mechanismen, die unter physiologischen Bedingungen jeden auftretenden Schleimhautdefekt zur Ausheilung bringen [8]. Die Ausschüttung von Neben-nierenrindenhormonen ist für die Sekretion sauren Magensaftes durch die Belegzellen und damit für die Ulcusgenese unerläßlich. Dies äußert sich z. B. klinisch in der Anazidität bei Morbus Addison und dem Wiederauftreten von Magensäure nach DOCA- [9] oder Cortison-Medikation [10]. Engel [10] und Gray [11] haben sogar Addisonfälle beschrieben, bei denen nach jahrelanger Cortisonbehandlung Ulcera aufgetreten sind. Diese medikamentös ausgelösten Ulcera erlauben Analogieschlüsse zur Erklärung der Pathogenese des gastro-duode-nalen Ulcus und verlagern das Ulcusproblem in den Hypothalamus, die Hypophyse und die Nebennierenrinde. Sind wir somit in unserer Erkenntnis über das «wie» der Ulcus-Genese wesentlich weiter gekommen, so stehen wir mit dem «warum» noch immer vor einem Rätsel. Die zur Ulcusentstehung führende Dysregulation kann kein Dauerzustand sein, sonst ware der periodische Verlauf des Ulcusleidens nicht zu erklären. Rene A. Gutmann [12] hat diesen periodischen Verlauf mit täglich auftretenden Be-schwerden während 3 bis 5 Wochen, abgelöst von monate- bis jahre-langen Intervallen von Beschwerdefreiheit, sogar zum diagnostischen Kriterium erhoben. ¥arum dieser periodische Verlauf, warum die Spontanremissionen innerhalb von wenigen Wochen, warum die fol-gende Beschwerdefreiheit und warum der neue Schub ? Es liegt nahe, jedes schubweise periodische Auftreten eines Leidens in irgend einer Weise mit dem rhythmischen Ablauf des Naturgeschehens in Zusam-menhang zu bringen. Mond und Planeten sind heute als pathogeneti-sche Einflußsphären wieder in die Feme gerückt. An ihre Stelle sind die Jahreszeiten gerückt und haben im medizinischen Denken und in den Texten der Lehrbüclier als Frühjahrs- und Herbstdisposition für das Auftreten eines Ulcusschubes einen gesicherten Platz errungen. Sind die Ulcusschübe tatsächlich saison-gebunden, oder handelt es sich um eine der unkontrolliert von Autor zu Autor übernommenen Konventionen ? Wer an einem Tag im April oder Oktober gleich drei Fälle von frisch einsetzendem Ulcusschub beobachten kann, wird 
damit ohne Zweifel die «Ulcussaison» als eröffnet betrachten. Aber kann ihm dasselbe nicht auch im Sommer oder Winter passieren ? Eine Nachprüfung drängte sich auf und war einfach durchzuführen.

Editorial

53

Im Zeitraum 1952 bis 1957 wurde bei 275 ambulanten Patienten mit gesichertem gastroduodenalen Ulcus (78 Ulcus ventriculi, 197 Ulcus duodeni) das jahreszeitliche Auftreten registriert. Dabei fiel auf, daß von den meisten Patienten, die bereits mit gestellter Diagnose vorausgegangener Schübe in Behandlung kamen, ein Frühjahr-Herbst-Rhythmus angegeben wurde. Wurde jedoch nur der gegen-wärtige, vom Untersucher selbst beobachtete Schub registriert, so änderte sich das Bild. In den meisten Fallen, besonders beim Ulcus duodeni, läßt sich der Beschwerdebeginn auf den Tag genau angeben. Nur diese Fälle wurden berücksichtigt, total 288 Ulcusschübe, ent-sprechend einem Monatsdurchschnitt von 24. Der Beschwerdebeginn verteilte sich auf die einzelnen Monate wie folgt: Januar 27, Februar 27, März 18, April 30, Mai 15, Juni 18, Juli 25, August 30, September 24, Oktober 27, November 33, Dezember 14. Es führt somit der November mit 33 vor April und August mit 30, Januar und Februar mit 27 und Juli mit 25, während den Schluß der Tabelle die Monate Dezember mit 14, Mai mit 15 und März und Juni mit 18 einnehmen. Auf die Jahreszeiten verteilen sich die Zahlen wie folgt: Frühjahr 63, Sommer 73, Herbst 84 und Winter 68. Wird nur der erste, selbst beobachtete Schub berücksichtigt, so erhält man: Frühjahr 7, Sommer 10, Herbst 11 und Winter 11. Differenzierung nach Geschlecht und Lokalisation des Ulcus in Magen oder Duodenum ergeben keine wesentlichen Abweichungen.

Diese Zahlen, erhoben an ambulanten Patienten einer gastroentero-logischen Praxis, sind zu klein, um daraus bindende Folgerungen zu ziehen. Aber sie zeigen doch, daß - zum mindesten in der beschriebe-nen Untersuchungsreihe - eine signifikante Frühjahrs- und Herbst-disposition nicht zum Ausdruck kommt, besonders nicht für das Frühjahr, das sowohl beim erstmaligen Auftreten wie bei den Rezidiv-fällen die geringste Zahl aufweist.

Mit Theoretisieren über die auslösende Ursache des Ulcusschubes kommen wir weniger weit als mit der Frage an den Patienten. In vier Fünftel der Fälle glaubt er, die auslösende Ursache zu kennen: Kummer, Sorgen, Ärger, enttäuschte Hoffnung, Überarbeitung, ge-spannte Situation, unregelmäßige Mahlzeiten oder Nikotinabusus. Dazu kommt in jüngster Zeit häufig die Medikation von ACTH, Cortison, Butazolidin oder Irgapyrin.

Zum Fortschritt in der Medizin gehört, daß durch kritische Be-obachtung der Einfluß unkontrollierbarer und damit unheimlicher Mächte und Naturgewalten mehr und mehr zurückgedrängt wird und 